\title{
Impact of prior smoking exposure and COPD comorbidity on treatment response to monoclonal antibodies in patients with severe asthma
}
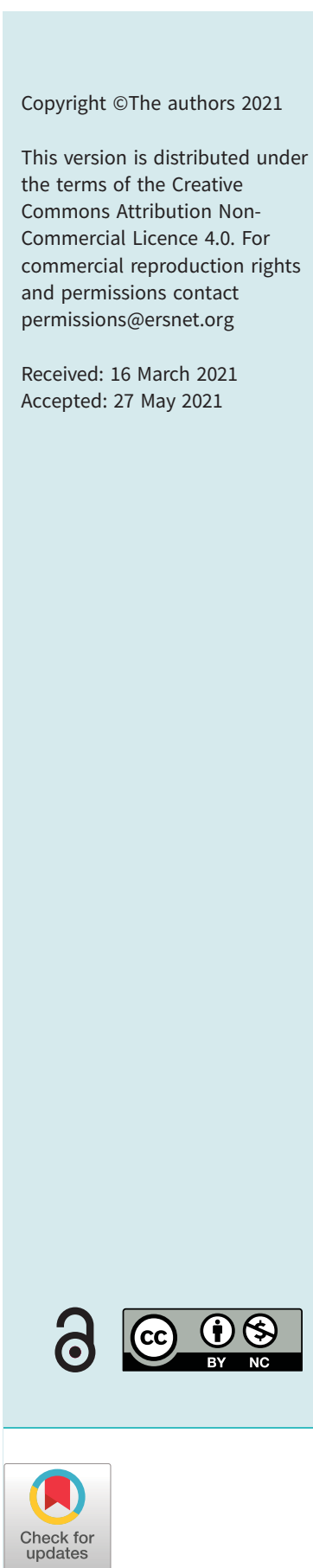

To the Editor:

Despite the prognostic role of smoking in asthma [1], clinical studies of asthma usually exclude current smokers or ex-smokers with a smoking history of $>10$ pack-years [2-5]. Specifically, the role of humanised monoclonal antibody therapy in patients with severe asthma and prior smoking exposure has not been studied; however, these drugs are used in patients with severe asthma and a history of smoking [6-9]. The aim of the present study was to evaluate in a real-world setting how a history of smoking and comorbid COPD affect the clinical outcome of patients suffering from severe asthma that are treated with monoclonal antibodies.

This is a single-centre, prospective and observational cohort study conducted at the Dept of Pneumology of the University Hospital Bonn (Bonn, Germany). Patients $(n=158)$ with severe asthma, based on Global Initiative for Asthma (GINA) recommendations [10] that now require antibody treatment, were included from November 2017 to April 2020. As suggested by the GINA recommendations, treatment was optimised to include smoking cessation 3 months before evaluation of the antibody treatment. Active smokers were excluded from the study. The study had the approval of the local ethics committee. We divided patients into two groups according to their history of smoking: >10 pack-years (ex-smokers) or less (nonsmokers). We evaluated the clinical response of patients to the newly initiated antibody therapies from baseline to follow-up after $6 \pm 3$ months on the therapy. We considered it to be a clinical improvement if the patient had an increase in the Asthma Control Test (ACT) score $\geqslant 4$ points [11], a decrease in the acute exacerbation rate of $50 \%$ [12] or improvement of lung function indicated by an increase of forced expiratory volume in $1 \mathrm{~s}\left(\mathrm{FEV}_{1}\right) \geqslant 12 \%$ or $\geqslant 200 \mathrm{~mL}$ [12]. In addition, non-contrast chest computed tomography (CT) scans were obtained with multidetector CT scanners ( $\geqslant 128$ rows) in 47 patients. Automated emphysema analysis was performed using commercially available software (IntelliSpace Portal; Philips Healthcare, Best, The Netherlands) in order to calculate the emphysema ratios. Lung parenchyma was considered emphysematous when it showed attenuation values of $<-950$ Hounsfield units at inspiration [13, 14]. An emphysema ratio was calculated for each CT dataset and was defined as the percentage of lung volume with emphysema divided by the total lung volume. Continuous variables were evaluated by using a paired t-test, categorical parameters by using Pearson's Chi-squared test and non-parametric values by using a Mann-Whitney U-test. A value of $\mathrm{p}<0.05$ was considered to be statistically significant.

Baseline clinical data for the patients $(n=158)$ are summarised in table 1 . All patients were on high-dose inhaled corticosteroids (mean \pm SD $1918 \pm 163$ versus $1890 \pm 176 \mu$ g beclomethasone dipropionate, deemed equivalent in the nonsmoking compared to the ex-smoking groups) and long-acting $\beta_{2}$-agonists, $95 \%$ were on long-acting muscarinic antagonists, while $65 \%$ of patients required oral corticosteroid (OCS) therapy. At baseline, clinical and laboratory parameters such as the exhaled nitric oxide fraction (mean $\pm S D$ $45.31 \pm 48.42 \mathrm{ppb}$ ), blood eosinophils (mean $\pm \mathrm{SD} \quad 492.16 \pm 382.86$ cells $\cdot \mu \mathrm{L}^{-1}$ ) and immunoglobulin $\mathrm{E}$ (mean \pm sD $557.26 \pm 828.20 \mathrm{IU} \cdot \mathrm{mL}^{-1}$ ) were similar between the two groups. The groups differed significantly in sex ( $74 \%$ versus $44 \%$ female in the nonsmoking compared to the ex-smoking group; $\mathrm{p}=0.010)$, age $(51 \pm 15$ years versus $59 \pm 11$ years; $\mathrm{p}=0.002)$ and pack-years $(5 \pm 2$ versus $28 \pm 17 ; \mathrm{p}<0.001)$. All patients received antibody therapies upon inclusion in the study, which did not differ between the two

Shareable abstract (@ERSpublications)

Patients suffering from severe asthma may benefit from an antibody treatment irrespective of their status as an ex-smoker https://bit.ly/3fYC8tC

Cite this article as: Morobeid H, Pizarro C, Biener L, et al. Impact of prior smoking exposure and COPD comorbidity on treatment response to monoclonal antibodies in patients with severe asthma. ERJ Open Res 2021; 7: 00190-2021 [DOI: 10.1183/23120541.00190-2021]. 


\begin{tabular}{|c|c|c|c|c|}
\hline & All & Ex-smokers & Nonsmokers & $\mathrm{p}$-value \\
\hline Patients & 158 & $48(30)$ & $110(70)$ & \\
\hline Females & $102(65)$ & $21(44)$ & $81(74)$ & $0.010^{\star}$ \\
\hline Age, years & $53.4 \pm 14.67$ & $58.88 \pm 11.40$ & $51.07 \pm 15.33$ & $0.002^{*}$ \\
\hline $\mathrm{BMI}, \mathrm{kg} \cdot \mathrm{m}^{-2}$ & $28.56 \pm 6.41$ & $28.90 \pm 4.89$ & $28.41 \pm 6.99$ & 0.060 \\
\hline Duration of the disease, years & $26.47 \pm 16.33$ & $24.42 \pm 18.65$ & $26.94 \pm 15.30$ & $0.045^{\star}$ \\
\hline Smoking pack-years & $21.70 \pm 18.07$ & $27.98 \pm 17.4$ & $4.94 \pm 1.83$ & $<0.001^{*}$ \\
\hline \multicolumn{5}{|l|}{ Comorbidities } \\
\hline COPD & $36(33)$ & $24(50)$ & $12(11)$ & $<0.001^{*}$ \\
\hline Emphysema & $19(12)$ & $10(21)$ & $9(8)$ & $0.017^{\star}$ \\
\hline Allergy & $92(84)$ & $27(56)$ & $66(60)$ & 0.331 \\
\hline Atopic dermatitis & $18(16)$ & $3(6)$ & $15(14)$ & 0.095 \\
\hline Chronic sinusitis/nasal polyps & $70(64)$ & $17(35)$ & $53(48)$ & 0.078 \\
\hline Obstructive sleep apnoea & $16(15)$ & $8(17)$ & $8(7)$ & 0.287 \\
\hline Gastro-oesophageal reflux & $11(10)$ & $3(6)$ & $8(7)$ & 0.891 \\
\hline Obesity & $17(15)$ & $3(6)$ & $14(13)$ & 0.107 \\
\hline \multicolumn{5}{|l|}{ ACT score } \\
\hline At baseline & $12.87 \pm 5.45$ & $10.78 \pm 4.02$ & $12.11 \pm 4.76$ & 0.353 \\
\hline At follow-up & $16.50 \pm 5.88$ & $16.13 \pm 6.03$ & $16.66 \pm 5.83$ & 0.568 \\
\hline$\Delta$ pre- to post-treatment & $4.07 \pm 5.71$ & $4.60 \pm 6.08$ & $3.83 \pm 5.55$ & 0.423 \\
\hline \multicolumn{5}{|l|}{$\mathrm{FEV}_{1}, \mathrm{~L}$} \\
\hline At baseline & $1.97 \pm 0.80$ & $1.66 \pm 0.60$ & $2.10 \pm 0.84$ & $0.007^{\star}$ \\
\hline At follow-up & $2.11 \pm 0.77$ & $1.86 \pm 0.68$ & $2.21 \pm 0.80$ & 0.263 \\
\hline$\Delta$ pre- to post-treatment & $0.14 \pm 0.42$ & $0.21 \pm 0.36$ & $0.12 \pm 0.46$ & 0.538 \\
\hline \multicolumn{5}{|l|}{ Exacerbation rate } \\
\hline At baseline & $4.08 \pm 4.16$ & $4.90 \pm 4.05$ & $3.73 \pm 4.17$ & 0.598 \\
\hline At follow-up & $0.22 \pm 0.63$ & $0.25 \pm 0.94$ & $0.21 \pm 0.43$ & 0.240 \\
\hline$\Delta$ pre- to post-treatment & $-3.89 \pm 4.12$ & $-4.79 \pm 4.14$ & $-3.49 \pm 4.06$ & 0.518 \\
\hline \multicolumn{5}{|c|}{ Regular oral corticosteroid dose, $\mathrm{mg} \cdot \mathrm{day}^{-1}$} \\
\hline At baseline & $7.15 \pm 8.32$ & $8.33 \pm 7.45$ & $6.62 \pm 8.67$ & 0.700 \\
\hline At follow-up & $2.35 \pm 4.76$ & $3.50 \pm 5.48$ & $1.84 \pm 4.33$ & 0.170 \\
\hline$\Delta$ pre- to post-treatment & $-3.91 \pm 8.74$ & $-3.79 \pm 10.35$ & $-3.97 \pm 7.97$ & 0.200 \\
\hline
\end{tabular}

patient groups (29\% omalizumab, 32\% benralizumab, 25\% dupilumab, 14\% mepolizumab, 1\% reslizumab). 18 patients were excluded from the analysis because they dropped out before reaching 4 months of treatment (12 in the nonsmoking and six in the ex-smoking group). Of these, 12 discontinued treatment owing to a lack of clinical improvement (nine in the nonsmoking versus three in the ex-smoking group), four owing to a lack of tolerability (two from each group) and two patients were lost to clinical follow-up (one from each group). Following the initiation of antibody treatment, overall asthma control improved significantly, with an increase of the ACT score $\geqslant 4$ points in $71 \%$ of the patients. Furthermore, an $89 \%$ reduction in the annualised exacerbation rate was achieved and a relevant improvement of lung function was seen in 38\% of cases. OCS consumption decreased by $67 \%$, and $68 \%$ of patients no longer required OCS. Again, these parameters were similar between the two groups (table 1). Single response criteria were fulfilled in all 158 cases (100\%), and all criteria were fulfilled in 42 cases (27\%). The quantification of emphysema by CT showed that ex-smoking patients had a significantly higher emphysema ratio, corresponding clinically to a rate of 50\% COPD comorbidity in the ex-smoking group. Again, there was no difference in the treatment responses in patients with emphysema and/or COPD. In addition, there was no correlation between the emphysema score and changes in ACT ( $\mathrm{r}=0.070$; $\mathrm{p}=0.640$ ), exacerbation rate $(\mathrm{r}=-0.041 ; \mathrm{p}=0.782)$, OCS use $(\mathrm{r}=0.075 ; \mathrm{p}=0.615)$ or $\mathrm{FEV}_{1}$ increase $(\mathrm{r}=-0.212$; $\mathrm{p}=0.153)$.

This is, to our knowledge, the first clinical observational study about the association between smoking history and the responsiveness of patients with severe asthma to GINA treatment step 5 add-on antibody therapy. It is well known that cigarette smoking is common in adults with asthma and is associated with increased morbidity and mortality [1]. The recent SHARP (Severe Heterogeneous Asthma Research collaboration, Patient-centred) study on patients with severe asthma documents a smoking history in 
Europe between $10.8 \%$ and $41.3 \%$. In Germany, $2.4 \%$ of such patients are current smokers and $37.8 \%$ are ex-smokers, with an average of 12.5 pack-years overall. However, the effects of treatment were not analysed in the SHARP study [9]. The treatment response found in our study is comparable to real-world data [6-8], which already showed patient responses in the real-world are similar to those in randomised controlled trials (although our study had a more stringent patient selection) [6-8]. In our study, ex-smokers with severe asthma benefited similarly compared to nonsmokers with severe asthma, in all of the selected end-points. The proportion of men in the ex-smoker group was higher despite the higher proportion of women seen across clinical trials and registries of patients with asthma, which demonstrates that smoking is still more common in males. The ex-smoker patient group was also significantly older compared to the nonsmoker group. Smoking is the major factor in the development of COPD, and differentiating between patients with asthma and COPD can be difficult; we used the new GINA/Global Initiative for Chronic Obstructive Lung Disease (GOLD) recommendations from 2020 for the diagnosis of asthma-COPD overlap (ACO) [10]. Accordingly, 50\% of ex-smokers suffering from severe asthma fulfilled the clinical criteria for the diagnosis of ACO. Of interest, this subgroup of patients with COPD comorbidity, in addition to the patients with emphysema, also showed a similar response to the antibody treatments.

Our study had both strengths and limitations. The strengths included a clinically detailed characterisation of prospectively enrolled patients with severe asthma, a detailed documentation of the response to newly prescribed antibody treatments, the low number of patients who were lost to follow-up and the "real-world" setting of the study. Limitations included the small sample size, the short follow-up interval and the registry nature of the data source, which obviously does not reach the same quality as a randomised clinical trial.

In conclusion, this real-world study extends previously published reports on the response of patients with severe asthma to antibody treatments, particularly by including patients with a history of smoking. We found that antibody treatments, when added to standard asthma therapies, are as efficacious in ex-smokers suffering from severe asthma as they are in nonsmokers, by improving the asthma control, exacerbation rate and lung function of these patients. In conclusion, our data suggest that patients suffering from severe asthma should benefit from antibody treatment, irrespective of their history of smoking. However, further placebo-controlled studies in this patient collective are warranted.

\section{Heba Morobeid ${ }^{1,4}$, Carmen Pizarro ${ }^{1,4}$, Leonie Biener $\oplus^{1}$, Gudrun Ulrich-Merzenich ${ }^{2}$, Daniel Kütting ${ }^{3}$, Georg Nickenig ${ }^{1}$ and Dirk Skowasch ${ }^{1}$}

${ }^{1}$ Dept of Internal Medicine II - Pneumology/Cardiology, University Hospital Bonn, Bonn, Germany. ${ }^{2}$ Dept of Internal Medicine III - Oncology, University Hospital Bonn, Bonn, Germany. ${ }^{3}$ Dept of Radiology, University Hospital Bonn, Bonn, Germany. ${ }^{4}$ These authors contributed equally.

Corresponding author: Dirk Skowasch (Dirk.Skowasch@ukbonn.de)

Acknowledgements: We thank Meghan Lucas (scientific coordinator in the Heart Center Bonn, University Hospital Bonn, Bonn, Germany) for proofreading the manuscript.

Data availability: All individual deidentified participant data (including data dictionaries), as well as additional, related documents, will be available immediately, upon request.

Conflict of interest: H. Morobeid has nothing to disclose. C. Pizarro reports personal fees from AstraZeneca and Novartis during the conduct of the study, and personal fees from AstraZeneca outside the submitted work. L. Biener has nothing to disclose. G. Ulrich-Merzenich reports grants from Novartis during the conduct of the study and outside the submitted work. D. Kütting has nothing to disclose. G. Nickenig has nothing to disclose. D. Skowasch reports personal fees from AstraZeneca, Novartis and GSK during the conduct of the study and outside the submitted work.

References

1 Thomson NC. Asthma and smoking-induced airway disease without spirometric COPD. Eur Respir J 2017; 49: 1602061.

2 Hanania NA, Wenzel S, Rosén $\mathrm{K}$, et al. Exploring the effects of omalizumab in allergic asthma: an analysis of biomarkers in the EXTRA study. Am J Respir Crit Care Med 2013; 15: 804-811. 
3 Bleecker ER, FitzGerald JM, Chanez P, et al. Efficacy and safety of benralizumab for patients with severe asthma uncontrolled with high-dosage inhaled corticosteroids and long-acting $\beta_{2}$-agonists (SIROCCO): a randomised, multicentre, placebo-controlled phase 3 trial. Lancet 2016; 388: 2115-2127.

4 Ortega HG, Liu MC, Pavord ID, et al. Mepolizumab treatment in patients with severe eosinophilic asthma. N Engl J Med 2014; 371: 1198-1207.

5 Castro M, Corren J, Pavord ID, et al. Dupilumab efficacy and safety in moderate-to-severe uncontrolled asthma. N Engl J Med 2018; 378: 2486-2496.

6 Harrison T, Canonica GW, Chupp G, et al. Real-world mepolizumab in the prospective severe asthma REALITI-A study: initial analysis. Eur Respir J 2020; 56: 2000151.

7 Kavanagh JE, Hearn AP, Dhariwal J, et al. Real-world effectiveness of benralizumab in severe eosinophilic asthma. Chest 2021; 159: 496-506.

8 Bousquet J, Humbert M, Gibson PG, et al. Real-world effectiveness of omalizumab in severe allergic asthma: a meta-analysis of observational studies. J Allergy Clin Immunol Pract 2021; 9: 2702-2714.

9 van Bragt JJMH, Adcock IM, Bel EHD, et al. Characteristics and treatment regimens across ERS SHARP severe asthma registries. Eur Respir J 2020; 55: 1901163.

10 Global Initiative for Asthma (GINA). Global Strategy for Asthma Management and Prevention. 2020. Available from: http://ginasthma.org

11 Schatz M, Kosinski M, Yarlas AS, et al. The minimally important difference of the Asthma Control Test. J Allergy Clin Immunol 2009; 124: 719-723.e1.

12 Drick N, Seeliger B, Welte T, et al. Anti-IL-5 therapy in patients with severe eosinophilic asthma - clinical efficacy and possible criteria for treatment response. BMC Pulm Med 2018; 18: 119.

13 Fischer AM, Varga-Szemes A, van Assen M, et al. Comparison of artificial intelligence-based fully automatic chest CT emphysema quantification to pulmonary function testing. AJR Am J Roentgenol 2020; 214: 1065-1071.

14 Herth FJF, Slebos DJ, Shah PL, et al. Protocol of a randomized controlled study of the PneumRx endobronchial coil system versus standard-of-care medical management in the treatment of subjects with severe emphysema (ELEVATE). Respiration 2019; 98: 512-520. 